\title{
Are more risk averse agents more optimistic? Insights from a rational expectations model
}

\author{
Elyès Jouini* and Clotilde Napp ${ }^{\dagger}$
}

June 20,2007

\begin{abstract}
We analyse the link between optimism and risk aversion in a model with endogeneous beliefs formation. We consider a model of partially revealing, competitive rational expectations equilibrium with diverse information, in which the distribution of risk aversion across individuals is unknown. We show that when a high individual level of risk-aversion is taken as a signal for a high average level of risk aversion, more risk averse agents are more optimistic. Such a positive correlation is important for the analysis of collective decision making as shown by Jouini and Napp (2007).
\end{abstract}

JEL Numbers: G12; D53; D82

Keywords: Optimism; risk aversion; rational expectations; heterogenous beliefs

\section{Introduction}

In equilibrium models with beliefs heterogeneity, it is shown that the belief of the representative agent is given by the average of the individual beliefs weighted by the individual risk tolerances (Jouini-Napp, 2007). It is then particularly important to investigate the nature of the link, if any, between individual risk aversion and beliefs. Our aim in this paper is to analyse if there is some correlation between these characteristics in a model with endogeneous beliefs formation.

For this purpose, we consider a model of partially revealing, competitive rational expectations equilibrium with diverse information. More precisely, we analyse a "large" market with a continuum of traders who posess diverse pieces of private information about the risky asset's payoff as in e.g. Grossman (1976), Hellwig (1980), Genotte-Leland (1980), Diamond-Verrecchia (1981), Admati (1985), Kyle (1989). Contrarily to standard models, we suppose that agents do not know the distribution of risk aversion in the economy. Private information is aggregated and, due to the noise on the distribution of risk aversion, is only partially revealed by the equilibrium prices. The equilibrium prices together with the private information, create the beliefs held by agents in equilibrium.

Assuming an imperfect knowledge of the aggregate risk aversion level is not an unrealistic assumption since the individual (or collective) risk aversion is in general hard to estimate and the estimations strongly depend on the chosen methodology (lotteries as in Donkers et al. 2001, option prices as in Jackwerth, 2000,

\footnotetext{
${ }^{*}$ Ceremade, Université Paris-Dauphine and Institut universitaire de France, Place du Maréchal de Lattre de Tassigny, 75 775, Paris cedex 16, jouini@ceremade.dauphine.fr

${ }^{\dagger}$ CNRS-DRM, Université Paris-Dauphine and CREST, Place du Maréchal de Lattre de Tassigny, 75 775, Paris cedex 16, clotilde.napp@dauphine.fr
} 
etc.). As underlined by Jackwerth (2000), "estimating risk aversion functions directly is still notoriously difficult". As a consequence, "there is no consensus in the literature on a precise aggregate risk aversion level" (Brennan-Torous, 1999). Furthermore, many models consider state dependent preferences (rankdependent utility, relative consumption utility, habit formation, recursive utility) hence state dependent risk aversion levels; the knowledge of the individual level of risk aversion would then suppose a continuous time monitoring of the individual choices and risk attitudes.

We prove existence results for such equilibria and we obtain closed form solutions for linear equilibria, thereby providing a theoretical rational expectations framework for the study of the link between individual beliefs and risk aversion. The question under consideration is the following. Are more risk averse agents more optimistic? More optimistic refers to a higher posterior expected payoff of the risky asset. We start from a model in which individual risk aversions and individual beliefs are independent. If the average risk aversion is a common knowledge, we show that there is no correlation between optimism and risk aversion at the equilibrium. On the contrary, if we suppose that a low (resp. high) individual level of risk aversion is taken as a private signal for a low (resp. high) average level of risk aversion then a positive correlation between optimism and risk aversion emerges at the equilibrium. The intensity of this correlation depends on the dispersion of the individual levels of risk aversion. We also analyse the extent to which risk-sharing schemes are modified.

The paper is organized as follows. Section 2 presents the model and the equilibrium concept. In Section 3, existence results as well as closed-form solutions are provided. Section 4 analyses the correlation between risk aversion and optimism as well as the risk-sharing schemes.

\section{The model}

We shall use the following version of Admati (1985)'s model. There is a continuum of agents, indexed by $i \in[0,1]$ and 2 periods denoted by 0 and 1 . Agents trade at date 0 and consume at date 1 . Each agent $i$ is endowed with an initial wealth $w_{0 i}$ and one unit of a given risky asset whose payoff at date 1 is $\widetilde{X} \sim \mathcal{N}\left(m_{x}, \sigma_{x}^{2}\right)$ (in terms of consumption good). There is also a riskless asset with zero interest rate. Each agent $i$ has a CARA utility function $u_{i}(w)=-\exp -a_{i} w$. We suppose that $\widetilde{a}: i \rightarrow a_{i}$ is measurable.

The information $I_{i}$ of agent $i$ consists of the equilibrium price and of his private information. As in standard models, agent $i$ 's private information first consists of the observation of a signal $\widetilde{Y}_{i}=\widetilde{X}+\widetilde{\varepsilon_{i}}$,

where $\widetilde{\varepsilon_{i}} \sim \mathcal{N}\left(0, \sigma_{\varepsilon}^{2}\right), \widetilde{\varepsilon_{i}}$ and $\widetilde{X}$ are independent, and $\widetilde{\varepsilon_{i}}$ is independent of $\widetilde{\varepsilon_{j}}, j \neq i$. We assume, as in Admati (1985) a "law of large numbers", i.e., that $\int_{0}^{1} \widetilde{\varepsilon_{i}} d i=0$.

Note that this model can account for diverse information as well as diverse opinion. Indeed, it is equivalent to assume that each agent has a signal and that the signals are randomly drawn around the true payoff or that each agent has an opinion (modeled by a belief conditional to the observation of a private signal) and that these opinions are also randomly drawn around the true payoff. In the differential information framework individuals care about others' beliefs because they contain information (others private signals). In the heterogenous opinions framework, individuals care about others' opinion if everyone thinks that the average opinion is closer to the truth than any individual opinion (conformity, informational social influence).

The distribution of risk aversion among agents is unknown; each agent only knows his own level of 
risk aversion. In standard rational expectations models, the unique meaningful risk aversion parameter at the equilibrium is the representative agent risk aversion, which is given by the harmonic average of the individual levels of risk aversion. We denote by $z \equiv\left(\int_{0}^{1} a_{i}^{-1} d i\right)^{-1}$ the (harmonic) average risk aversion. We suppose that $z$ is unknown and modeled by a random variable $\widetilde{Z}$. This randomness can be interpreted in two ways leading to two possible specifications. The first interpretation is that there are econometric estimations of the average level of risk aversion that are available and common knowledge. We suppose then that agents agree on an exogenous distribution ${ }^{1}$ of $\widetilde{Z} \sim \mathcal{N}\left(m_{z}, \sigma_{z}^{2}\right)$ where $\sigma_{z}^{2}$ measures the confidence level of these estimations. In this case, agents private information only consists of the private signal since agents do not infer any information from their own observed level of risk aversion. The second interpretation is that individual and collective risk aversion levels are difficult to estimate, in particular because these parameters may be state dependent and may evolve through time. In that case, agents rely on their own level of risk aversion in order to estimate the average level of risk aversion; they interpret their own level of risk aversion as private information on the average level of risk aversion. We do not specify a particular form for the agents prior on the risk aversion distribution and the learning process. We simply assume that the posterior distribution of $\widetilde{Z}$ following the observation of the individual level of risk aversion $a_{i}$ satisfies $\widetilde{Z} \mid a_{i} \sim \mathcal{N}\left(a_{i}, \sigma_{z}^{2}\right)$. The normality condition on the posterior distribution of $\widetilde{Z}$ is, in particular, satisfied if we assume that agents have a prior distribution of risk tolerance $\frac{1}{\widetilde{a}} \sim \mathcal{N}\left(\frac{1}{z}, \frac{\sigma^{2}}{z^{2}}\right)$ with $z \sim \mathcal{N}\left(m, \tau^{2}\right)$. For $\tau=\infty$ (diffuse prior on $Z$ ), the posterior distribution of $\widetilde{Z}$ is normal with mean ${ }^{2}$ $a_{i}$.

Note that the considered distribution $\widetilde{Z} \mid a_{i}$ is a posterior distribution in the sense that it is posterior to the observation by each agent of his own level of risk aversion. However this distribution is prior to the observation of prices. Hence we are not assuming that each agent considers his own level or risk aversion as actually driving market risk aversion nor that his risk aversion represents a large portion of the market risk aversion. This would be incompatible with the fact that each agent is atomistic. We are just assuming that, without any knowledge about others' levels of risk aversion, each agent enters the market with a distribution that is only based on his own level of risk aversion.

We suppose that the objective distribution of risk aversion in the economy as well as the subjective posterior belief $\widetilde{Z} \mid a_{i}$ are independent of $\widetilde{X}$, and $\widetilde{\varepsilon_{i}}$ and that all the introduced random variables have a jointly normal distribution.

Since in the large economy, each agent is "small" and the private signals are independently distributed, the particular realizations of these signals should have no effect on the realized equilibrium price. We will therefore search for linear equilibrium prices that only depend on "market aggregates" $\widetilde{X}$ and $\widetilde{Z}$. The following definition is standard.

Definition 1. A linear equilibrium for the economy is defined by a price $\widetilde{p}$ and demand functions $\left(\widetilde{\theta}_{i}\right)_{i \in[0,1]}$ such that (a) $\widetilde{p}$ is of the form $\alpha+\beta \widetilde{X}+\gamma \widetilde{Z} ;$ (b) $\widetilde{\theta}_{i} \in \arg \max E_{i}\left[u_{i}\left(\widetilde{w_{1 i}}\right)\right]$ with $\widetilde{w_{1 i}}=$ $w_{0 i}+\theta_{i} \widetilde{X}+\left(1-\theta_{i}\right) \widetilde{p} ;(c) \int_{0}^{1} \widetilde{\theta}_{i} d i=1$.

\footnotetext{
${ }^{1}$ In fact, $\widetilde{Z}$ can only be positive, but it is standard in financial economics, for easily understandable reasons of tractability, to suppose a normal distribution, with a "reasonably small" variance.

${ }^{2}$ We have obtained similar results assuming that $\widetilde{Z} \mid a_{i} \sim \mathcal{N}\left(k a_{i}-\ell, \sigma_{z}^{2}\right)$ with $k \in[0,1]$ and $\ell \geq 0$. Indeed, it could be natural to consider each $a_{i}$ as the individual estimation of the arithmetic average of the individual levels of risk aversion. We have then to take into account that the harmonic average $z$ is smaller than the arithmetic average.
} 


\section{Existence of equilibria}

When agents agree on an exogenous distribution of $\widetilde{Z} \sim \mathcal{N}\left(m_{z}, \sigma_{z}^{2}\right)$ and do not infer any information from their own level of risk aversion, it is easy to prove that there exists a unique linear equilibrium price. It is of the form $\widetilde{p}=\left(1-\beta_{e}\right) m_{x}+\beta_{e} \widetilde{X}-V_{e} \widetilde{Z}$, where $V_{e}$ is the ex-post variance $\operatorname{Var}\left(\tilde{X} \mid I_{i}\right)$. Note that this variance does not involve the signal value. It is known ex-ante and is common to all the individuals. It is also easy to check that, as expected, $\beta_{e}$ decreases with $\sigma_{\varepsilon}^{2}$. Furthermore, when $\sigma_{z}^{2} \rightarrow \infty$, the price becomes uninformative and $\beta_{e}$ converges to $\frac{\sigma_{x}^{2}}{\sigma_{x}^{2}+\sigma_{\varepsilon}^{2}}$ which corresponds, as expected, to the weight of $\widetilde{X}$ in the Walrasian equilibrium. Finally, $\beta_{e}$ decreases when $\sigma_{x}^{2}$ increases and when the relative precision of the signal $\frac{\sigma_{x}^{2}}{\sigma_{\varepsilon}^{2}}$ is kept constant, which is also natural.

Let us assume now that the beliefs on $\widetilde{Z}$ are endogeneously specified.

Theorem 1. If $\widetilde{Z} \mid a_{i} \sim \mathcal{N}\left(a_{i}, \sigma_{z}^{2}\right)$ and if $\sigma_{\varepsilon}^{2} \sigma_{z}^{2} \geq 4$ then there exists a linear equilibrium. It is of the form $\widetilde{p}=\alpha+\beta \widetilde{X}+\gamma \widetilde{Z}$ with $\gamma<0$ and $\beta>0$.

Proof. Note first that the conditional distribution of $\widetilde{X}$ given $\widetilde{Y}_{i}, \alpha+\beta \widetilde{X}+\gamma \widetilde{Z}$ and $a_{i}$ is the same as the conditional distribution of $\widetilde{X}$ given $\widetilde{Y}_{i}$ and $\widetilde{p} \equiv \alpha+\beta \widetilde{X}+\gamma \widetilde{Z}$ with $\widetilde{Z} \sim \mathcal{N}\left(a_{i}, \sigma_{z}^{2}\right)$. Then we easily obtain that the conditional distribution of $\widetilde{X}$ given $I_{i}$ is also normal with mean and variance of the form

$$
E_{i}[\tilde{X}]=m_{x}+C\left(y_{i}-m_{x}\right)+D\left(\beta\left(x-m_{x}\right)+\gamma\left(z-a_{i}\right)\right), \quad V \equiv \operatorname{VAR}_{i}[\tilde{X}]=\sigma_{x}^{2}(1-C-\beta D)
$$

with $(C, D)=\left(\frac{\gamma^{2} \sigma_{z}^{2} \sigma_{x}^{2}}{\gamma^{2} \sigma_{x}^{2} \sigma_{z}^{2}+\beta^{2} \sigma_{x}^{2} \sigma_{\varepsilon}^{2}+\gamma^{2} \sigma_{z}^{2} \sigma_{\varepsilon}^{2}}, \frac{\beta \sigma_{x}^{2} \sigma_{\varepsilon}^{2}}{\gamma^{2} \sigma_{x}^{2} \sigma_{z}^{2}+\beta^{2} \sigma_{x}^{2} \sigma_{\varepsilon}^{2}+\gamma^{2} \sigma_{z}^{2} \sigma_{\varepsilon}^{2}}\right)$.

As in the standard setting with exponential utility functions and normal distributions, the portfolio optimization problem leads to $\widetilde{\theta}_{i}=\frac{E_{i}[\widetilde{X}]-\widetilde{p}}{a_{i} \operatorname{VAR}_{i}[\widetilde{X}]}$. There exists then a linear equilibrium if and only if $\gamma=$ $-\sigma_{x}^{2}(1-\beta)$ and $C+D \beta=\beta$. For $\sigma_{x} \sigma_{\varepsilon}>0$, this system admits a solution if and only if $Q(\beta)=$ $\sigma_{\varepsilon}^{2}\left(\sigma_{\varepsilon}^{2}+\sigma_{x}^{4} \sigma_{z}^{2}+\sigma_{x}^{2} \sigma_{z}^{2} \sigma_{\varepsilon}^{2}\right) \beta^{2}-\left(2 \sigma_{x}^{2}+\sigma_{\varepsilon}^{2}\right) \sigma_{z}^{2} \sigma_{\varepsilon}^{2} \sigma_{x}^{2} \beta+\sigma_{x}^{4} \sigma_{z}^{2} \sigma_{\varepsilon}^{2}$ admits roots in $(0,1)$. For $\sigma_{\varepsilon}^{2} \sigma_{z}^{2} \geq 4, Q$ has two roots $0<\beta^{-}<\beta^{+}<1$. We will see in the next section that only one of the two roots, namely $\beta^{-}$, has good properties and is sensible.

The equilibrium price for the risky asset is then an increasing function of $\widetilde{X}$, its payoff, and a decreasing function of $\widetilde{Z}$, the average level of risk aversion. When the price of the risky asset increases, an individual is uncertain whether it is the result of an increase in the risky asset payoff or whether, on average, everyone is less risk averse. The observation of a given price should lead to an update of the distributions of $\widetilde{X}$ and $\widetilde{Z}$ in two opposite directions.

The ex-post expected value $E_{i}[\tilde{X}]$ increases with the private signal $\widetilde{Y}_{i}$, with the equilibrium price $\widetilde{p}$ and with the level of risk aversion $a_{i}$ whereas $\operatorname{VAR}_{i}[\tilde{X}]$ is independent of $a_{i}$ as well as of the realizations of $\tilde{Y}_{i}$ and $\widetilde{p}$. This means that all other things being equal, the distribution of $\widetilde{X}$ conditional to the information $I_{i}$ increases with the level of risk aversion in the sense of the first stochastic dominance.

In fact, we have $\widetilde{p}=(1-\beta) m_{x}+\beta \widetilde{X}-V \widetilde{Z}$ and this price corresponds to the equilibrium price in a Walrasian setting where agent $i$ has a belief on $\tilde{X}$ given by $\tilde{X} \mid I_{i}$. The realization $p$ is of the form

\footnotetext{
${ }^{3}$ This condition is analogous to those in Glosten (1989) or Bhattacharya and Spiegel (1992). It means that there is enough noise in the economy. This assumption may be dropped if we assume that $\widetilde{Y}_{i}=\widetilde{X}+\widetilde{U}+\widetilde{\varepsilon_{i}}$ where $\widetilde{U}$ is an additional noise common to all the agents with $\widetilde{U} \sim \mathcal{N}\left(0, \sigma_{u}^{2}\right)$ and $\sigma_{u}>0$.
} 
$p=\left(\int a_{i}^{-1} E_{i}(\tilde{X}) d i\right) /\left(\int a_{i}^{-1} d i\right)-V z$ and corresponds to the equilibrium price in an economy with homogenous beliefs in which the common belief would be given by the average of the individual beliefs weighted by the individual levels of risk tolerance.

Finally, what is the right value for $\beta$ ? When the price becomes noninformative, i.e. when $\sigma_{z}^{2} \rightarrow \infty$,

we have $\beta^{+} \rightarrow 1$ and $\beta^{-} \rightarrow \frac{\sigma_{x}^{2}}{\sigma_{\varepsilon}^{2}+\sigma_{x}^{2}}$. The natural value is $\frac{\sigma_{x}^{2}}{\sigma_{\varepsilon}^{2}+\sigma_{x}^{2}}$. It corresponds to a Walrasian equilibrium in which each agent only uses the private signal. The value of $\beta^{+}=1$ corresponds to the situation in which information is fully revealed. The $\operatorname{root} \beta^{-}$has then a more natural asymptotic behavior than $\beta^{+}$.

We can also prove that $\beta^{-}$decreases when the precision of individual information $1 / \sigma_{\varepsilon}^{2}$ decreases (with $\sigma_{x}^{2}$ and $\sigma_{z}^{2}$ constant). It also decreases when the total noise $\sigma_{x}^{2}$ increases (with $\frac{\sigma_{\varepsilon}^{2}}{\sigma_{x}^{2}}$ and $\sigma_{z}^{2}$ remain constant). It increases when the total noise $\sigma_{x}^{2}$ increases (with $\sigma_{\varepsilon}^{2}$ and $\sigma_{z}^{2}$ constant). Finally, $\beta^{-}$decreases when the total noise $\sigma_{z}^{2}$ increases (with $\sigma_{\varepsilon}^{2}$ and $\sigma_{x}^{2}$ constant).

The coefficient $r^{-}=\frac{\left|\gamma^{-}\right|}{\beta^{-}}$increases when $\sigma_{x}^{2}$ increases (with $\frac{\sigma_{\varepsilon}^{2}}{\sigma_{x}^{2}}$ and $\sigma_{z}^{2}$ constant): the relative weight of the average level of risk aversion $\widetilde{Z}$ with respect to the weight of the payoff of the risky asset $\widetilde{X}$ increases with the uncertainty on $\widetilde{X}$.

Finally, $\left|\gamma^{-}\right|$decreases with $\sigma_{\varepsilon}^{2}$ (all other things being equal), with $\sigma_{z}^{2}$ (all other things being equal), with $\sigma_{x}^{2}$ (when $\frac{\sigma_{\varepsilon}^{2}}{\sigma_{x}^{2}}$ remain constant) and with $\sigma_{x}^{2}$ (all other things being equal).

All these properties are natural and consistent with Hellwig (1980) or Diamond and Verrecchia (1981). Conversely $\beta^{+}$and $\gamma^{+}$have systematically the opposite monotonicity properties.

\section{Equilibrium Properties}

In our model, individual risk aversion and individual prior belief are independent. Does the interaction between agents induce a correlation between individual (posterior) belief and individual risk aversion? In the exogeneous case, it is easy to obtain that risk aversion and posterior belief are independent. In the endogeneous case, we have seen that the agent individual posterior belief increases with the level of risk aversion in the sense of the first stochastic dominance. There is then a positive correlation between optimism and risk aversion. Analytically, if we denote by $\bar{a}=\int a_{i} d i$ the average risk aversion, the covariance between posterior belief (or more precisely the expected posterior payoff) and risk aversion is given by $\int_{0}^{1} E_{i}[\tilde{X}]\left(a_{i}-\bar{a}\right) d i=V D \operatorname{VAR}_{i}\left(a_{i}\right)>0$.

The intuition for this positive correlation is simple. In the model with an endogenous specification of the average level of risk aversion, for a given equilibrium price, which, as we have seen, increases with the payoff of the risky asset and decreases with the average level of risk aversion, a more risk averse agent will perceive a higher average level of risk aversion and, by observing a given equilibrium price, will infer a higher expected payoff.

To what extent does an endogenous specification of the average level of risk aversion modify the risk sharing schemes ? The average of agent $i$ optimal demand over the different possible signals is given by $\int \theta_{i} d \varepsilon_{i}=D+(1-D) \frac{z}{a_{i}}$. It is decreasing in $a_{i}$ (recall that $\left.D \leq 1\right)$ which is natural; a more risk averse agent will have a lower optimal demand in the risky asset.

The average (over all possible signals) optimal of agent $i$ in the exogeneous (reference) case is equal to $\frac{z}{a_{i}}$ and we would have obtained the same value in the Walras setting. There is then less risk-sharing in the endogenous setting. The dispersion of the level of risk aversion has a weaker impact in the endogenous 
setting, in the sense that the less risk averse insure less than in the reference setting. In the reference situation, on average, a relatively very risk averse (resp. risk tolerant) individual will have very low (resp. very high) optimal demand. In the endogenous setting, the very risk averse becomes more optimistic, which raises his optimal demand, and the very risk tolerant agent becomes more pessimistic, which reduces his optimal demand. As a result, there is less dispersion in the optimal demands.

\section{Conclusion}

We have shown that the non observability of others level of risk aversion leads to a positive correlation between optimism and risk aversion. Indeed, the equilibrium price increases with the payoff of the risky asset and decreases with the average level of risk aversion. Hence, more risk averse agents perceive an equilibrium price that seems too high and infer from there high expectations on the asset's payoff.

This positive correlation between optimism and risk aversion leads the less risk averse to insure less than in the standard setting and induces less risk sharing among agents.

It is interesting to note that a positive correlation between optimism and risk aversion has been empirically observed in a purely behavioral setting by Ben Mansour et al. (2007). In this last paper, the authors show that agents exhibit pessimism and that more risk averse agents are more optimistic.

Such a positive correlation is particularly important from the collective decision making point of view as shown by Jouini and Napp (2007). It induces a pessimistic bias in the representative agent belief that is represented by a risk tolerance weighted average of the individual beliefs. It may also contribute to a better understanding of the risk premium puzzle.

\section{References}

Admati, A. R., 1985. A noisy rational expectations equilibrium for multi asset securities markets. Econometrica, 53, 629-657.

Ben Mansour, S., Jouini, E., Marin, J.-M., Napp, C. and C. Robert, 2006. Are more risk averse agents less pessimistic? A Bayesian estimation approach. Unpublished working paper.

Bhattacharya, U. and M. Spiegel, 1992. Insiders, outsiders and market breakdowns. Review of Financial Studies, 4, 255-282.

Brennan, M.J. and W.N.Torous, 1999. Individual Decision Making and Investor Welfare, Economic Notes, $28,119-143$.

Diamond, D. W. and R.E. Verrecchia, 1981. Information aggregation in a noisy rational expectations economy. Journal of Financial Economics, 9, 221-235.

Donkers, B., Melenberg, B. and A. Van Soest, 2001. Estimating Risk attitudes Using Lotteries: a Large Sample Approach. Journal of Risk and Uncertainty, 22, 165-195.

Genotte, G. and H. Leland, 1990. Market Liquidity, Hedging, and Crashes. American Economic Review, 80, 999-1021.

Glosten, L.R., 1989. Insider Trading, Liquidity, and the Role of the Monopolist Specialist. Journal of Business, 62, 211-235. 
Grossman, S., 1976. On the efficiency of competitive stock markets where agents have diverse information. Journal of Finance, 31, 573-585.

Hellwig, M. F., 1980. On the aggregation of information in competitive markets. Journal of Economic Theory, 22, 477-498.

Jackwerth, J. C., 2000. Recovering Risk Aversion from Option Prices and Realized Returns. Review of Financial Studies, 13(2), 433-51.

Jouini, E., and C. Napp, 2007. Consensus consumer and intertemporal asset pricing with heterogenous beliefs. Review of Economic Studies. To appear.

Kyle, A. S., 1989. Informed Speculation with Imperfect Competition. Review of Economic Studies, 56, $317-356$. 